\title{
Statistical models for point-counting data
}

\author{
Pieter Vermeesch \\ Department of Earth Sciences \\ University College London \\ p.vermeesch@ucl.ac.uk
}

August 4, 2018

\begin{abstract}
Point-counting data are a mainstay of petrography, micropalaeontology and palynology. Conventional statistical analysis of such data is fraught with problems. Commonly used statistics such as the arithmetic mean and standard deviation may produce nonsensical results when applied to point-counting data. This paper makes the case that point-counts represent a distinct class of data that requires different treatment. Point-counts are affected by a combination of (1) true compositional variability and (2) multinomial counting uncertainties. The relative significance of these two sources of dispersion can be quantified by a chi-square statistic and -test. For datasets that pass the chi-square test for homogeneity, the 'pooled' composition is shown to represent the optimal estimate for the underlying population. It is obtained by simply adding together the counts of all samples and normalising the resulting values to unity. However, more often than not, point-counting datasets fail the chi-square test. The overdispersion of such datasets can be captured by a random effects model that combines a logistic normal population with the usual multinomial counting uncertainties. This gives rise to the concept of a 'central' composition as a more appropriate way to average overdispersed data. Two- or three-component datasets can be displayed on radial plots and ternary diagrams, respectively. Higher dimensional datasets may be visualised and interpreted by Correspondence Analysis (CA). This is a multivariate ordination technique that is similar in purpose to Principal Component Analysis (PCA). CA and PCA are both shown to be special cases of Multidimensional Scaling (MDS). Generalising this insight to multiple datasets allows point-counting data to be combined with other data types such as chemical compositions by means of 3-way MDS. All the techniques introduced in this paper have been implemented in the provenance R-package, which is available from http://provenance.london-geochron.com.
\end{abstract}




\section{Introduction}

The mineralogical composition of silicilastic sediments can be determined by tallying the occurrence of various minerals in a representative sample of (200-400, say) grains (Dryden, 1931; Van der Plas and Tobi, 1965; Weltje, 2002). Similarly, the fossil content of a deep sea sediment core may be characterised by tabulating the relative abundances of various species among >100 randomly selected specimens (Patterson and Fishbein, 1989; Buzas, 1990; Fatela and Taborda, 2002). Or palaeobiological environments may be reconstructed by tabulating the relative frequency of different types of pollen in a palaeosol or charcoal (Barkley, 1934; Clark, 1982; Weng et al., 2006).

These are all examples of multivariate counting experiments, in which the unknown proportions of different species of minerals, fossils or pollen are estimated by counting a finite number of randomly selected items from a representative sample. Despite the widespread use of this type of data in the Earth Sciences and related fields, their statistical analysis is demonstrably underdeveloped.

For example, there currently exists no agreed method to average multi-sample point-counting datasets, or to quantify point-counting data dispersion. Traditionally, these operations were done by taking the arithmetic mean and standard deviation, respectively. Unfortunately, this may easily produce nonsensical results. For example, Weltje (2002) shows that the common practice of using '2-sigma' confidence bounds around the arithmetic mean can produce physically impossible negative values when applied to petrographic point-counts.

To solve these problems, Weltje (2002) argues that point-counts should be treated as compositional data, which are defined as "vectors representing parts of a whole that only carry relative information" (PawlowskyGlahn and Buccianti, 2011). According to this definition, compositional data can be renormalised to a constant sum (e.g., 100\% if the composition is expressed as percentages, or 1 if fractions are used) without loss of information. Aitchison $(1982,1986)$ shows that the statistical analysis of such data is best carried out using a simple logratio transformation.

To illustrate this approach, let $\left\{a_{i}, b_{i}, c_{i}\right\}$ be a three-component dataset, where $a_{i}+b_{i}+c_{i}=1$ for $1 \leq i \leq m$. Then this dataset can be mapped to a bivariate Euclidean data space as follows:

$$
u_{i}=\ln \left(a_{i} / c_{i}\right) \text { and } v_{i}=\ln \left(b_{i} / c_{i}\right)
$$


After performing the desired statistical analysis (such as calculating averages and confidence regions) on the transformed data $\left\{u_{i}, v_{i}\right\}$, the results can be mapped back to the ternary diagram by means of an inverse logratio transformation:

$$
\begin{aligned}
a_{i} & =\frac{\exp \left[u_{i}\right]}{\exp \left[u_{i}\right]+\exp \left[v_{i}\right]+1}, \\
b_{i} & =\frac{\exp \left[v_{i}\right]}{\exp \left[u_{i}\right]+\exp \left[v_{i}\right]+1}, \text { and } \\
c_{i} & =\frac{1}{\exp \left[u_{i}\right]+\exp \left[v_{i}\right]+1}
\end{aligned}
$$

This procedure yields geologically meaningful (geometric) means and confidence regions. Weltje (2002)'s adoption of logratio statistics to point-counting data represents a huge improvement over the 'crude' statistics employed previously. But it does not solve all our problems. There are two crucial differences between point counts and the classical compositional data discussed by Aitchison $(1982,1986)$.

First, point-counting data are associated with significant (counting) uncertainties, which are ignored by classical compositional data analysis. For a single sample, this uncertainty is adequately described by multinomial counting statistics (Section 6 of Weltje, 2002). But for larger datasets comprised of multiple samples, existing procedures to construct confidence regions (as discussed in Section 7 of Weltje, 2002) are inadequate because they lump together the 'observational' dispersion caused by counting statistics and the true 'geological' dispersion. Bloemsma and Weltje (2015) describe a method to disentangle these two sources of uncertainty in a logratio context. They show that deconvolution of (spectroscopic) count data into a scale vector and a proportions matrix significantly improves multivariate analysis.

Second, point-counting data often contain zero values, which are incompatible with the log-ratio transformation defined in Equation 1. This problem also applies to the aforementioned approach by Bloemsma and Weltje (2015). These authors circumvented the occurrence of sporadic zeros by replacing them with small positive numbers. This and alternative 'imputation' strategies are further discussed by Martín-Fernández et al. (2003). When the number of zeros is small, imputation is considered to have a minimal influence on the data covariance structure. However, some point-counting datasets are dominated by zeros. So the presence of such values is not a cosmetic problem, but a fundamental characteristic of this particular data type. The statistical treatment of point-counting data needs to address this issue at a deeper level. 
The present paper solves these long standing problems using established statistical methods adopted from other disciplines. Much of the paper is based on the work of Galbraith (2005) in fission track geochronology. The fission track method is based on the ratio of the number of spontaneous ${ }^{238} \mathrm{U}$-tracks to the number of neutron-induced ${ }^{235} \mathrm{U}$-tracks per unit area in accessory minerals such as apatite or zircon. This is equivalent to a simple two-component point-counting problem. Section 2 uses this equivalence to derive the concept of a 'pooled composition'. We will show that the latter represents the most reliable (in terms of accuracy and precision) average of homogeneous point-counting data.

The analytical uncertainty of individual point-counting proportions may greatly vary between samples. Section 3 introduces Galbraith (1988)'s radial plot as a graphical means of visualising such 'heteroscedastic' data. Originally developed for fission track data, the radial plot can also be used to display point-counting ratios, which frequently occur in the Earth Sciences. Radial plots allow a visual assessment of the degree to which counting uncertainties can explain the observed scatter between multiple ratio estimates. Section 4 presents a formal statistical test to make this assessment more quantitative.

The pooled composition is only applicable to samples that pass this chi-square test for sample homogeneity. Multi-sample datasets that fail the chi-square test are said to be 'overdispersed' with respect to the counting uncertainties. The degree of overdispersion may be quantified by means of a continuous mixture model (Section 5). This model leads to the concept of a 'central composition' as a better alternative to the pooled composition of Section 2. Section 6 generalises the continuous mixture model from two to three (or more) components.

Finally, Section 7 introduces Correspondence Analysis (CA) as a useful ordination technique for multivariate point-counting data. CA is closely related to compositional Principal Component Analysis (PCA). But unlike the latter method, it does not suffer from the zero counts problem.

All the techniques discussed above will be illustrated with a combination of synthetic and real examples. The methods of Sections 2-6 will use the two datasets shown in Table 1. Data 1 consists of 20 random samples of 23-94 items each, which were drawn from a discrete trinomial distribution with $45 \%$ of component $a, 45 \%$ of component $b$ and $10 \%$ of component $c$. Data 2 comprises a further 20 samples that were drawn from a continuous distribution whose mode is the same as that of Data 1 , but which adds $100 \%$ of dispersion around this mode. 
Thus, Data 2 has two sources of dispersion (counting error and true population dispersion), whereas Data 1 only has one (counting error). Note that both datasets contain fewer counts per sample than is customary in real world applications. But they are nevertheless realistic if we consider them to be ternary subcompositions of higher dimensional datasets.

\begin{tabular}{c|ccc|c||c|ccc|c}
\multicolumn{9}{c||}{ Data 1} & \multicolumn{5}{c}{ Data 2} \\
\hline$\#$ & $a$ & $b$ & $c$ & $R$ & $\#$ & $a$ & $b$ & $c$ & $R$ \\
\hline 1 & 16 & 18 & 4 & 38 & 1 & 23 & 24 & 5 & 52 \\
2 & 25 & 17 & 3 & 45 & 2 & 60 & 24 & 7 & 91 \\
3 & 18 & 18 & 0 & 36 & 3 & 45 & 43 & 12 & 100 \\
4 & 7 & 14 & 3 & 24 & 4 & 2 & 53 & 4 & 59 \\
5 & 12 & 10 & 3 & 25 & 5 & 8 & 32 & 10 & 50 \\
6 & 32 & 30 & 13 & 75 & 6 & 53 & 21 & 23 & 97 \\
7 & 35 & 38 & 13 & 86 & 7 & 1 & 6 & 3 & 10 \\
8 & 20 & 20 & 7 & 47 & 8 & 2 & 17 & 1 & 20 \\
9 & 10 & 9 & 3 & 22 & 9 & 10 & 10 & 4 & 24 \\
10 & 29 & 36 & 5 & 70 & 10 & 2 & 35 & 3 & 40 \\
11 & 34 & 34 & 9 & 77 & 11 & 29 & 21 & 3 & 53 \\
12 & 22 & 47 & 12 & 81 & 12 & 2 & 13 & 0 & 15 \\
13 & 9 & 9 & 2 & 20 & 13 & 3 & 9 & 0 & 12 \\
14 & 37 & 36 & 13 & 86 & 14 & 34 & 1 & 0 & 35 \\
15 & 46 & 25 & 16 & 87 & 15 & 28 & 19 & 4 & 51 \\
16 & 50 & 37 & 7 & 94 & 16 & 49 & 11 & 3 & 63 \\
17 & 28 & 34 & 8 & 70 & 17 & 0 & 72 & 2 & 74 \\
18 & 39 & 50 & 6 & 95 & 18 & 55 & 28 & 13 & 96 \\
19 & 44 & 36 & 10 & 90 & 19 & 7 & 8 & 3 & 18 \\
20 & 28 & 21 & 4 & 53 & 20 & 20 & 5 & 2 & 27 \\
\hline$C$ & 541 & 539 & 142 & $N=1222$ & $C$ & 433 & 452 & 90 & $N=987$
\end{tabular}

Table 1: Two synthetic ternary point-counting datasets. Data 1 was drawn from a single multinomial distribution with population proportions of $45 \%, 45 \%$ and $10 \%$ for components $a, b$ and $c$, respectively. Data 2 was drawn from a continuous mixture of multinomial distributions whose true proportions were drawn from a bivariate logistic normal distribution with a geometric mean of $45 \%$ for $a$ and $b, 10 \%$ for $c$, and $100 \%$ dispersion with a correlation coefficient of -0.5 between the two logratio dimensions. $R, C$ and $N$ refer to the row, column, and total sums, respectively.

\section{The pooled composition}

Let us partition a population of objects (such as minerals, fossils or pollen) into two classes. Let $\theta$ be the true fraction of the first class, and $(1-\theta)$ the true fraction of the second class, respectively. Suppose that we have collected $m$ representative samples of randomly selected items from this population. For each of these samples, let $n_{i 1}$ represent the number of items belonging to the first class and $n_{i 2}$ the number of items belonging to 
the second class (for $1 \leq i \leq m$ ). Further let $n_{i}$ be the total number of counts in the $i^{\text {th }}$ sample so that $n_{i} \equiv n_{i 1}+n_{i 2}$. The probability of observing $n_{i 1}$ and $n_{i 2}$ given $n_{i \bullet}$ then follows a binomial distribution:

$$
p\left(n_{i 1}, n_{i 2} \mid n_{i \bullet}\right)=\left(\begin{array}{c}
n_{i} \\
n_{i 1}
\end{array}\right) \theta^{n_{i 1}}(1-\theta)^{n_{i 2}}
$$

The true value of $\theta$ is unknown but can be estimated (as $\hat{\theta}$ ) by jointly considering all $m$ samples and maximising their summed log-likelihood $\left(\mathcal{L}_{p}\right)$ :

$$
\begin{aligned}
\mathcal{L}_{p} & =\sum_{i=1}^{m}\left[\ln \left(\begin{array}{c}
n_{\bullet} \\
n_{i 1}
\end{array}\right)+n_{i 1} \ln (\theta)+n_{i 2} \ln (1-\theta)\right] \\
& =\text { constant }+n_{\bullet 1} \ln (\theta)+n_{\bullet 2} \ln (1-\theta)
\end{aligned}
$$

where $n_{\bullet 1} \equiv \sum_{i=1}^{m} n_{i 1}$ and $n_{\bullet 2} \equiv \sum_{i=1}^{m} n_{i 2}$. Equation 4 is maximised if $\hat{\theta}=n_{\bullet 1} /\left(n_{\bullet 1}+n_{\bullet 2}\right)$. In other words, the maximum likelihood solution is found by pooling all the counts together. A 100(1- $\alpha) \%$ confidence interval for $\theta$ is given by:

$$
\frac{n_{\bullet 1}}{n_{\bullet 1}+\left(n_{\bullet 2}+1\right) F_{2(n \bullet 2+1), 2 n \bullet 1}^{\alpha}}<\theta<\frac{\left(n_{\bullet 1}+1\right) F_{2(n \bullet 1+1), 2 n \bullet 2}^{\alpha}}{n_{\bullet 2}+\left(n_{\bullet}+1\right) F_{2\left(n_{\bullet}+1\right), 2 n \bullet 2}^{\alpha}}
$$

where $F_{a, b}^{\alpha}$ denotes the 100(1- $\left.\alpha\right)$-percentile of an F-distribution with $a$ and $b$ degrees of freedom. The same equation can be used to construct a confidence interval for $\theta_{i}$ by replacing $n_{\bullet 1}$ with $n_{i 1}$ and $n_{\bullet 2}$ with $n_{i 2}$.

Let us consider the binary subcomposition comprised of $a$ and $b$ in samples 11 and 13 of Data 1 as an example. For sample $11, a=b=34$ so that $\hat{\theta}=0.5 \pm 0.11$. For sample $13, a=b=9$ so that $\hat{\theta}=0.5 \pm 0.21$. In other words, although the binomial parameter estimates of these two samples are the same, the width of their $95 \%$ confidence intervals differ by a factor of two. In statistical terms, this is called heteroscedasticity. The following section of this paper will introduce a graphical means of visualising such data.

\section{A radial plot for point-counting ratios}

In many Earth Sciences applications, it is not so much the absolute proportions but the ratios between binary (sub)compositions that are of interest. For example, in the context of heavy mineral analysis the apatite/tourmaline, monazite/zircon, and $\mathrm{TiO}_{2}$ /zircon ratios (e.g., Morton and Hallsworth, 1994), and the epi- 
dote/garnet ratio (Heroy et al., 2003) have all be used to indentify the provenance of sediments. In micropalaeontology, the ratio of benthic to planktonic foraminifera has been used as a productivity index (e.g., Berger and Diester-Haass, 1988). And in palynology, the arboreal/non-arboreal pollen ratio is widely used as an index of landscape openness (e.g., Herzschuh, 2007).

Given an estimate of the binomial parameter, $\hat{\theta}$, such ratios can be simply obtained as $\hat{\theta} /(1-\hat{\theta})$. The corresponding 100(1- $\alpha) \%$ confidence intervals can be calculated using Equation 5. Applying this to samples 11 and 13 of Data 1 as an example, we obtain $a / b$-ratio estimates of $1.00+0.53 /-0.35$ for for sample 11 , and 1.00 $+1.43 /-0.58$ for sample 13. So like the binomial parameter estimates, also the ratio estimates are heteroscedastic. A radial plot is a graphical device that was specifically designed to display such data.

Given a set of paired counts $\left\{n_{i 1}, n_{i 2}\right\}$ (for $1 \leq i \leq m$ ), the radial plot is a scatter diagram that sets out $\left(z_{i}-z_{\circ}\right) / s_{i}$ against $1 / s_{i}$, where

$$
\begin{aligned}
& z_{i}=\arcsin \left(\sqrt{\left(n_{i 1}+3 / 8\right) /\left(n_{i \bullet}+3 / 4\right)}\right), \\
& z_{\circ}=\arctan \left(\sqrt{n_{\bullet 1} / n_{\bullet 2}}\right), \text { and } \\
& s_{i}=1 /\left(2 \sqrt{n_{i \bullet}+1 / 2}\right)
\end{aligned}
$$

Precise measurements plot towards the right hand side of this plot and imprecise measurements to the left. The actual $n_{1 i} / n_{2 i}$-ratio is proportional to the slope of a line connecting the $i^{\text {th }}$ data point to the origin. The corresponding values are shown on a circular scale shown at some convenient radial distance away from the origin.

Figure 1.i shows a radial plot for the first two components $(a$ and $b$ ) of Data 1. Samples 11 and 13 of Data 1 have been highlighted. Recall that the estimated $a / b$-ratio of sample 11 has the same value but twice the precision of sample 13. Therefore, sample 11 plots at the same angle, but towards the right of sample 13.

Figure 1.ii displays the $a / b$-ratios of Data 2. Let us have a closer look at samples 2 and 7 , which are marked in black on this diagram. The total number of grains counted in these two samples are $n_{2} \bullet=60+24=84$, and $n_{7 \bullet}=1+6=7$, respectively (Table 1 ). So sample 2 is twelve times larger than sample 7 , allowing a more precise $a / b$-ratio estimate. Sample 2 therefore plots to the right of sample 7 , causing the $95 \%$-confidence interval 
The dispersion of the data with respect to the counting uncertainties can be visually assessed by comparing the vertical scatter of the data with a confidence band of two standard errors wide drawn on either side of the origin. If approximately $95 \%$ of the data plot within this interval, then the data are compatible with a homogeneous composition. This is the case for Data 1, which can therefore be safely averaged using the pooled ratio (Figure 1.i). In contrast, the $a / b$-ratios of Data 2 significantly scatter beyond the '2-sigma' confidence region (Figure 1.ii). In this case the pooled average should be abandoned in favour of a heterogeneous model (Section 5).

In conclusion, the radial plot is a useful device to visually assess the dispersion of point-counting ratios. The next section of this paper introduces a formal statistical test to make this assessment more quantitative.

i. central ratio $=1.00 \pm 0.14$
MSWD $=1.4, \mathrm{p}\left(\chi^{2}\right)=0.13$
pooled ratio $=1.00 \pm 0.10$
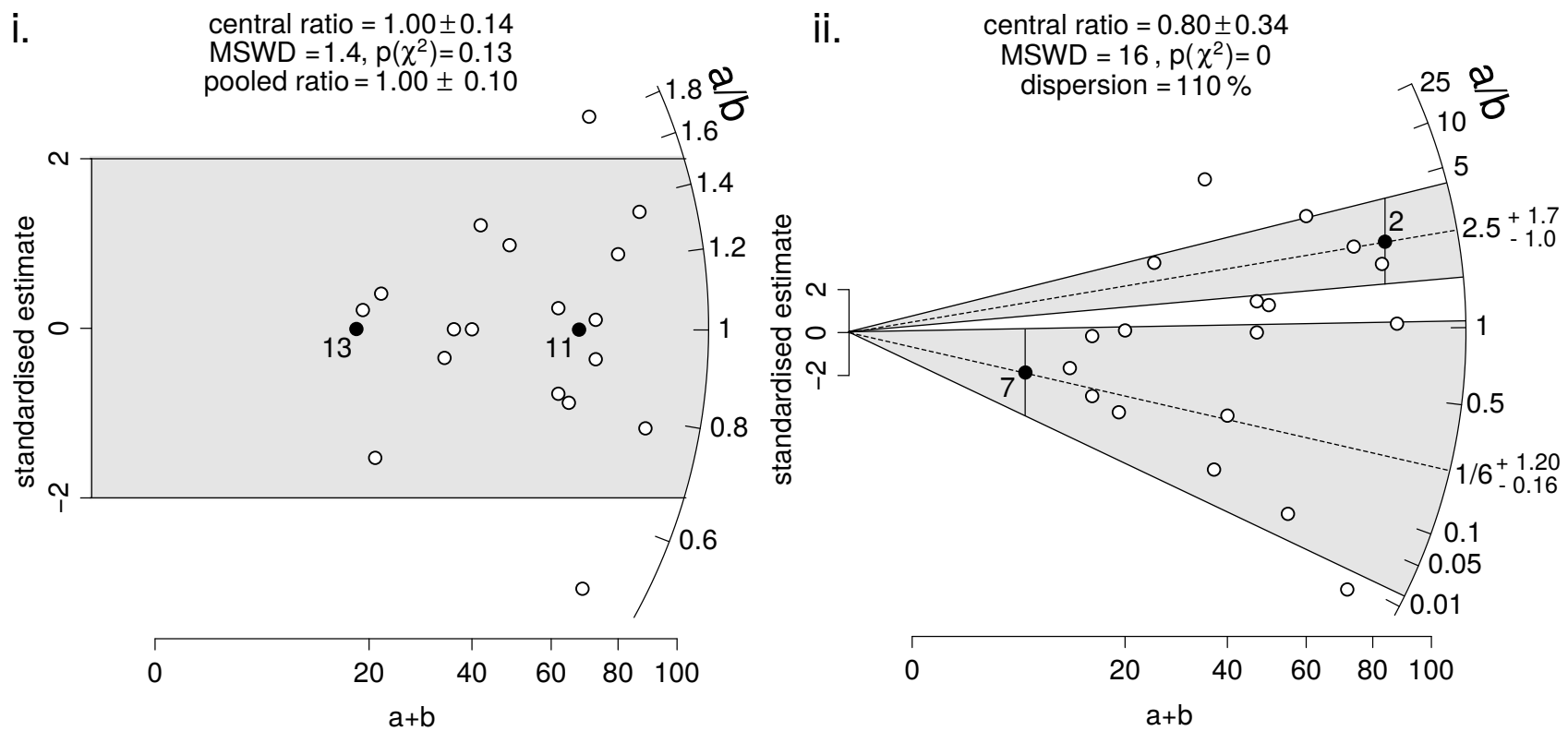

Figure 1: Radial plots of the binary subcompositions $a$ and $b$ for Table 1. i) approximately $95 \%$ of the samples in Data 1 plot within a symmetric '2-sigma' band around the origin. These data are therefore compatible with a single homogeneous population. The pooled composition then is the best estimate for the average composition (Section 2). ii) the $a / b$-ratio of each sample can be obtained by projecting the corresponding scatter point onto the radial scale. The black dots mark samples 2 (right) and 7 (left) of Data 2. Projecting a '2-sigma' error bar onto the same scale yields the $95 \%$ confidence intervals of $a / b=2.5+1.7 /-1.0$ for sample 2 and $a / b=0.17+1.20 /-0.16$ for sample 7 , respectively. In contrast with Data 1 , the $a / b$-ratios of Data 2 do not fit within a '2-sigma' band. In this case the data are more adequately described by a random effects model with two parameters: the central ratio and the dispersion. 


\section{A chi-square test for compositional homogeneity}

In order for the pooled composition to be a meaningful description of the detrital population, all samples must be derived from a single true composition. In other words, any observed differences in the $n_{i 1} / n_{i 2}$-ratios must be due to binomial counting statistics alone. The validity of this assumption can be verified by calculating the chi-square statistic:

$$
\chi_{\text {stat }}^{2}=\frac{1}{n_{\bullet 1} n_{\bullet}} \sum_{i=1}^{m} \frac{\left[n_{i 1} n_{\bullet 2}-n_{i 2} n_{\bullet}\right]^{2}}{n_{i \bullet}}
$$

If $\chi_{\text {stat }}^{2}$ is greater than the $100(1-\alpha)$-percentile of a chi-square distribution with $(m-1)$ degrees of freedom, then the null hypothesis of compositional homogeneity is rejected on a $100(1-\alpha) \%$ confidence level, where $\alpha$ is usually taken to be 0.05 .

An alternative way to quantify the dispersion of the data with respect to the expected counting fluctuations is to divide $\chi_{\text {stat }}^{2}$ by the number of degrees of freedom. This parameter is known as the 'reduced chi-square statistic', but is also frequently referred to as the 'Mean Square of the Weighted Deviates' in the Earth Sciences (Wendt and Carl, 1991):

$$
\mathrm{MSWD} \equiv \chi_{\text {stat }}^{2} /(m-1)
$$

If the observed scatter is entirely due to binomial counting statistics, then the MSWD is expected to take values close to unity. This is the case for Data 1, which is characterised by an MSWD of 1.4 and a p-value of 0.13 . The latter value is well above the 0.05 cutoff, making the pooled composition the most appropriate average. The pooled $a / b$-ratio of Data 1 is $1.00 \pm 0.10$, which agrees with the known ratio of 1.00 (Section 1 ).

MSWD values significantly greater than one indicate the presence of excess scatter beyond the binomial counting statistics. This is the case for Data 2, which yields an MSWD-value of 16 and a p-value close to zero. In this situation, the pooled average is not the most appropriate estimator for the population average and a more realistic model must be used. An example of one such model is given in Section 5 . 


\section{Continuous mixtures}

Datasets that fail the chi-square test for homogeneity are incompatible with a single binomial population. Instead their binomial population parameter $\theta$ may be drawn from a continuous distribution. Suppose that $\theta$ is drawn from a logistic normal distribution with geometric mean $\mu$ and coefficient of variation $\sigma$, and define

$$
\beta \equiv \ln \left(\frac{\theta}{1-\theta}\right) \sim \mathcal{N}\left(\mu, \sigma^{2}\right)
$$

where $\mathcal{N}\left(\mu, \sigma^{2}\right)$ stands for "the normal distribution with mean $\mu$ and variance $\sigma^{2}$ ". Note that $\beta$ is a logratio similar to those defined in Equation 1. Given the usual $m$ sets of point-counts $\left\{n_{i 1}, n_{i 2}\right\}$ and maximising the $\log$-likelihood function $\mathcal{L}_{c}$

$$
\mathcal{L}_{c}=\sum_{i=1}^{m} \ln \left\{\left(\begin{array}{l}
n_{i \bullet} \\
n_{i 1}
\end{array}\right) \int_{-\infty}^{\infty} \frac{\exp \left[\beta n_{i 1}\right]}{(\exp [\beta]+1)^{n_{i}}} \frac{\exp \left[-\frac{1}{2}\left(\frac{\beta-\mu}{\sigma}\right)^{2}\right]}{\sigma \sqrt{2 \pi}} d \beta\right\}
$$

yields two estimates $\hat{\mu}$ and $\hat{\sigma}$ whose approximate standard errors may be obtained by inverting the Hessian matrix of second derivatives of $\mathcal{L}_{c}$. The integrals in Equation 10 cannot be evaluated analytically, but a quick numerical solution is provided by Galbraith and Laslett (1993). The 'central' composition is then defined as:

$$
\hat{\theta}=\frac{\exp [\hat{\mu}]}{\exp [\hat{\mu}]+1}
$$

which is akin to the inverse logratio transformation defined in Equation 2. The central $a / b$-ratio for Data 2 is $0.80 \pm 0.34$ (Figure 1.ii), which again agrees with the true ratio of 1.00 that was reported in Section 1.

The dispersion estimate $\hat{\sigma}$ quantifies the geological variability of the underlying population. This is just as useful a quantity as the central value itself. It estimates the relative spread of the underlying population without the binomial counting errors. For example the coefficient of variation (standard deviation divided by mean) of the $a / b$-ratio measurements for Data 2 is $\sim 260 \%$. This is far greater than the $\sim 110 \%$ dispersion estimated by the random effects model. The latter estimate is much closer to the true dispersion of the underlying population, whose value is $100 \%$ (Section 1).

It is useful to note that, for samples that pass the chi-square test for sample homogeneity, the pooled ratio is the same as the central ratio. This is indeed the case for Data 1, which yields a pooled ratio of $1.00 \pm 0.10$ 
and a central ratio of $1.00 \pm 0.14$. The larger $95 \%$ uncertainty interval of the latter is due to the loss of one degree of freedom that is required to estimate $\hat{\sigma}$.

\section{Ternary models}

The statistical models presented in the previous sections can be generalised from two to three or more components. This is trivial for homogeneous populations such as Data 1, whose pooled composition is shown in Figure 2.i. For heterogeneous populations such as Data 2, the continuous mixture model of Section 5 can be generalised by defining two population parameters $\beta_{1} \equiv \ln \left[\theta_{1}\right]-\ln \left[1-\theta_{1}-\theta_{2}\right]$ and $\beta_{2} \equiv \ln \left[\theta_{2}\right]-\ln \left[1-\theta_{1}-\theta_{2}\right]$. Assuming that $\beta_{1}$ and $\beta_{2}$ are drawn from a bivariate normal distribution with mean $M$ and covariance matrix $\Sigma$, the three-component equivalent to Equation 10 becomes

$$
\begin{array}{r}
\mathcal{L}_{t}=\sum_{i=1}^{m} \ln \left\{\frac{n_{i \bullet} !}{n_{i 1} ! n_{i 2} ! n_{i 3} !} \int_{-\infty}^{\infty} \int_{-\infty}^{\infty} \frac{\exp \left[\beta_{1} n_{i 1}+\beta_{2} n_{i 2}\right]}{\left(\exp \left[\beta_{1}\right]+\exp \left[\beta_{2}\right]+1\right)^{n_{i}}}\right. \\
\left.\frac{\exp \left[-\frac{1}{2}(B-M)^{T} \Sigma^{-1}(B-M)\right]}{2 \pi \sqrt{|\Sigma|}} d \beta_{1} d \beta_{2}\right\}
\end{array}
$$

where $\left\{n_{i 1}, n_{i 2}, n_{i 3}\right\}$ are the ternary counts of the $i^{\text {th }}$ sample, with $n_{i 1}+n_{i 2}+n_{i 3}=n_{i \bullet}$ for $1 \leq i \leq m$;

$$
B=\left[\begin{array}{l}
\beta_{1} \\
\beta_{2}
\end{array}\right] ; M=\left[\begin{array}{l}
\mu_{1} \\
\mu_{2}
\end{array}\right] ; \text { and } \Sigma \equiv\left[\begin{array}{cc}
\sigma_{1}^{2} & \sigma_{1,2} \\
\sigma_{1,2} & \sigma_{2}^{2}
\end{array}\right]
$$

in which $\sigma_{1}$ and $\sigma_{2}$ are the standard deviations of $\beta_{1}$ and $\beta_{2}$, and $\sigma_{1,2}$ is their covariance. Equation 12, like Equation 10, does not have an analytical solution and requires numerical integration for each sample. The fast algorithm of Galbraith and Laslett (1993) can be used to estimate $\mu_{1}, \mu_{2}, \sigma_{1}$ and $\sigma_{2}$ so that only the covariance $\sigma_{1,2}$ remains to be found. The central composition is then estimated by substituting $\hat{\mu}_{1}$ for $u_{i}$ and $\hat{\mu}_{2}$ for $v_{i}$ in Equation 2. Figure 2.ii applies this model to Data 2, showing the central composition as a black square and using the dispersion estimate $\hat{\Sigma}$ to define a $95 \%$ confidence region for the underlying population (red line).

The definition of $\beta_{1}$ and $\beta_{2}$ that is used in Equation 12 is consistent with the logratio approach of Equation 1. However other parameterisations are possible as well. For example, we could define three logistic population parameters $\left(\beta_{i} \equiv \ln \left[\theta_{i}\right]-\ln \left[1-\theta_{i}\right]\right.$ for $\left.1 \leq i \leq 3\right)$ to ensure compatibility with the bivariate random effects 
model of Section 5. These alternative parameterisations are interchangeable with each other and can easily be converted to each other.

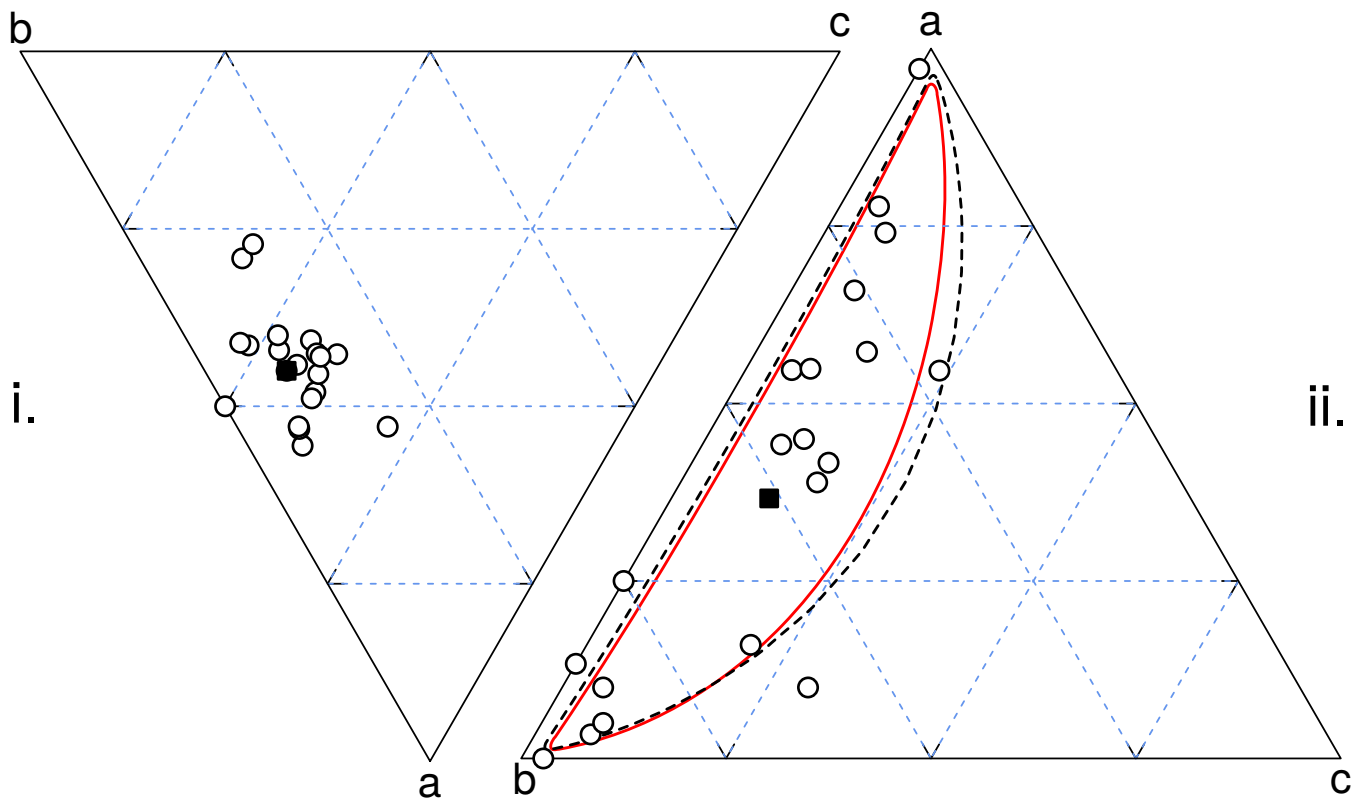

Figure 2: Statistical analysis of ternary point-counting data. i. Data 1 is sampled from a homogeneous population, whose mean is given by the pooled composition shown as a black square. ii. Data 2 is derived from a continuous mixture (see the caption of Table 1 for details). Its estimated central composition is shown as a black square, and the $95 \%$ confidence envelope corresponding to its dispersion parameters as a red contour line. The dashed black contour marks the true dispersion region for the population, also shown at $95 \%$ confidence.

\section{Correspondence analysis}

The previous sections of this paper have shown that binary or ternary datasets can be visualised as radial plots and ternary diagrams, respectively. These two-dimensional graphics are useful for interpreting point-counting data, but cannot so easily be applied to higher dimensional datasets. In this section, we will consider the general case of a $K$-component dataset $X$ contained in an $[m \times K]$-matrix. We will explore some strategies to display such a dataset as a two-dimensional graphic.

Principal Component Analysis (PCA, Pearson, 1901) is an ordination techniques that is commonly used for exploratory data analysis of multi-dimensional datasets. PCA is a two step process. First, the data are 'centred' by subtracting the arithmetic mean composition from each column. Second, the centred data are decomposed into an orthogonal set of $K$ principal components. Plotting the first two principal components against each 
other then yields the desired two-dimensional data projection.

Unfortunately, PCA cannot readily be applied to compositional data or point-counting data. This is because the first step involves taking an arithmetic mean, which we have already shown to be problematic in Section 1. Subjecting the data to a logratio transformation prior to PCA analysis solves this problem (Aitchison, 1983). But this solution generally does not work for point-counting data due to its inability to handle zero count data. This issue is aggravated by the tendency for high dimensional datasets to contain more zeros than lower dimensional datasets do.

Correspondence Analysis (CA, Greenacre, 1984) fixes these issues by explicitly treating the data as counts. CA is a multivariate ordination technique that is conceptually similar to PCA. To understand the relationship between the two methods, it is useful to point out that PCA and CA are both special case of another exploratory data analysis method called Multidimensional Scaling (MDS, Kruskal and Wish, 1978; Vermeesch, 2013). Given a table of pairwise 'dissimilarities' between samples, MDS produces a map in which similar samples plot close together and dissimilar samples plot far apart.

PCA is a special case of MDS in which the dissimilarities are Euclidean distances. CA is another special case of MDS in which the dissimilarities are chi-square distances (Legendre and Gallagher, 2001; Greenacre, 2005):

$$
d_{i j}=\sqrt{\sum_{k=1}^{K} \frac{X_{\bullet \bullet}}{X_{\bullet k}}\left(\frac{X_{i k}}{X_{i \bullet}}-\frac{X_{j k}}{X_{j \bullet}}\right)^{2}}
$$

where $d_{i j}$ is the dissimilarity between samples $i$ and $j$ (with $1 \leq i, j \leq m$ ); $X_{\bullet}=\sum_{i=1}^{m} X_{i k} ; X_{i \bullet}=\sum_{k=1}^{K} X_{i k}$; $X_{j} \bullet=\sum_{k=1}^{K} X_{j k}$; and $X_{\bullet \bullet}=\sum_{i=1}^{m} \sum_{k=1}^{K} X_{i k}$. In the case of PCA, the principal components are obtained by linear combination of the original variables. The weightings of these variables can be displayed together with the transformed data as a biplot (Aitchison and Greenacre, 2002). The same principle can be applied to CA (Figure 3). 


\section{Examples}

All the methods discussed in this paper were added to the provenance package of Vermeesch et al. (2016). Written in the statistical programming language $\mathrm{R}$, provenance comes with a query-based user interface that does not require any programming skills. Alternatively, the full functionality of the package can also be accessed via the command line, as demonstrated in the following tutorial.

Point-counting data can be read from a .csv file using the read.counts function. For example, to read the second dataset from Table 1:

data2 <- read. counts ('data2.csv')

Plotting the ratios of the first two variables $(a / b)$ as a radial plot (Figure 1.i):

radialplot (data2, num=' a ', den=' $b$ ' )

where num and den are optional arguments denoting the names of the numerator and denominator component, respectively. Plotting the full dataset on a ternary diagram and constructing its $95 \%$ confidence region (Figure 2.ii):

\# create a ternary data object:

tern $<-$ ternary (data2)

\# show the data on a ternary diagram

\# as white circles without data labels:

plot (tern, pch=1, labels=NA)

\# add the $95 \%$ confidence region:

ternary.ellipse (tern, alpha $=0.05)$

where everything that follows a hash character ('\#') is a comment and is ignored. Next, let us consider a real dataset of heavy mineral counts from Namibia published by Vermeesch et al. (2016). The following code snippet calculates the central composition for these data:

HM $<-$ read.counts ('HM. csv')

avg $<-$ central (HM)

The variable avg contains a $[5 \times 15]$ table in which each column corresponds to a mineral species, and the rows contain (1) the central value for the binomial parameters $\left(\theta_{i}\right.$ for $\left.1 \leq i \leq 15\right)$ for those minerals; (2) the 
standard error for the binomial parameters; (3) the overdispersion parameter for the binary composition parameter $\ln \left[\theta_{i}\right]-\ln \left[1-\theta_{i}\right] ;(4)$ the MSWD value for each binary subcomposition; and (5) the corresponding p-value.

Next, we will perform a correspondence analysis of the Namib data. But before doing so, it is important to point out that $\mathrm{CA}$ is most sensitive to the least abundant components. To mitigate the effects of this phenomenon, it is useful to pre-process the data. The following code snippet selects the most abundant minerals (epidote, garnet, amphibole and clinopyroxene) from the datasets and amalgamates the ultra-stable minerals (zircon, tourmaline and rutile), which have similar petrological significance:

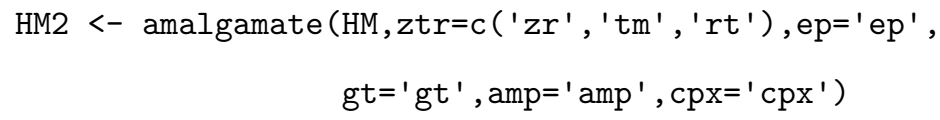

The resulting data object (HM2) still contains a number of zero values, but is no longer dominated by them. The actual CA calculation then proceeds as follows:

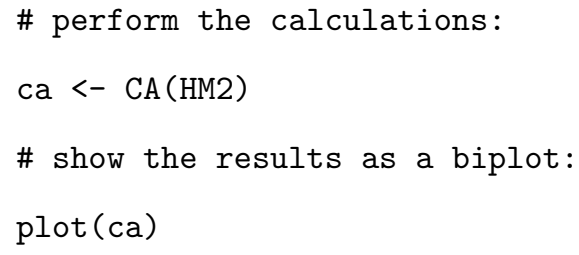

The biplot (Figure 3) displays the samples in black and the minerals as red arrows. The tight clustering of samples N1, N2, N3, N10, N12, N14, T8 and T13 reflects the compositional similarity between these samples, which were all derived from the coastal parts of the Namib Sand Sea (Vermeesch and Garzanti, 2015). In contrast, inland samples N4, N5, N8 and N9 plot elsewhere, indicating that they have a different composition. This is due to a combination of provenance and hydraulic sorting effects (Vermeesch and Garzanti, 2015).

The configuration of the mineral labels provides further insight into the factors that cause the dispersion of the samples on the biplot. For example, the orientation of the red arrows shows that samples N8 and N9 are enriched in garnet and ultra-stable minerals, whereas sample N5 is enriched in epidote relative to the coastal samples. The arrows for epidote and clinopyroxene point in opposite directions, indicating that these two minerals are anti-correlated with each other. In contrast, the arrow for garnet is perpendicular to that of epidote. This indicates that garnet and epidote are uncorrelated with each other. 


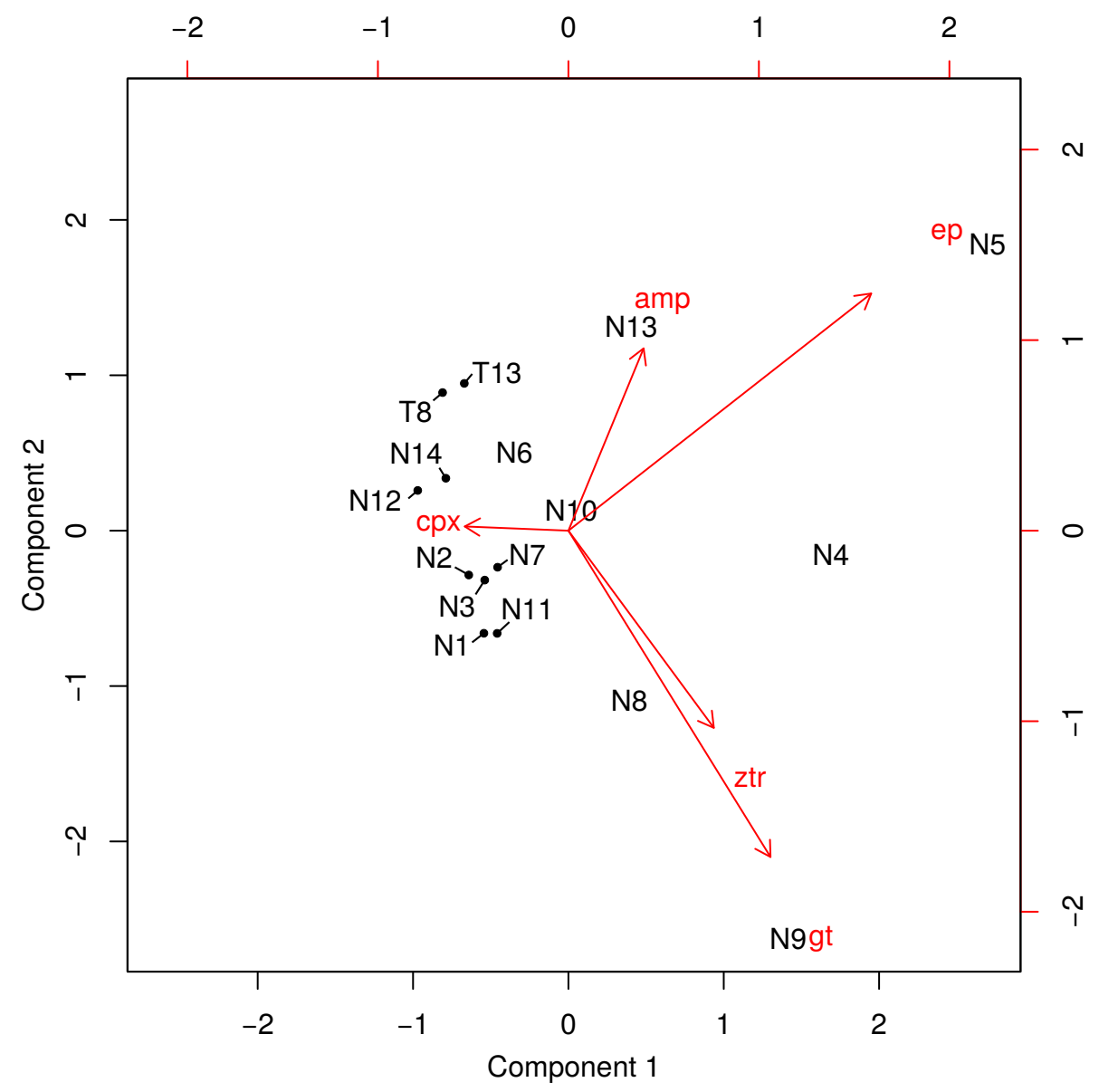

Figure 3: Correspondence analysis of heavy mineral compositions from Namibia (Vermeesch et al., 2016) shown as a biplot. Samples are shown in black, minerals in red.

\section{Discussion and conclusions}

It is common practice in sedimentary petrography, palaeontology and palynology to report the relative abundances of minerals, fossils or pollen as percentages. Unfortunately, by doing so one loses the ability to quantify the statistical uncertainty of the underlying point-counting data. Normalisation of point-counts also compromises the ability to deal with missing (zero) components.

The statistical methods reviewed in this paper are built on the recognition that point-counts represent a distinct class of data. This new data class shares aspects with, but is fundamentally different from Aitchison (1986)'s compositional data. Compositional data only carry relative information (Pawlowsky-Glahn and Buccianti, 2011), and the absolute abundances of their components are irrelevant. In stark contrast with this, for point-counting data the absolute abundances do matter, because they control the precision of the estimated 
compositions (Bloemsma and Weltje, 2015).

This observation leads to a first recommendation, which is to report the total number of counts for each sample in published data tables. This allows the recovery of the raw point-counting data. Those data can then be further analysed using the techniques introduced in this paper.

Compositional data and point-counting data are closely related to each other. In fact, point-counting data are underlain by compositional populations. These populations can be constrained using a combination of multinomial and logratio statistics.

If the data are underlain by a single, fixed composition, then the point-counting data follow a multinomial distribution. In this case, the fixed composition of the underlying population can be estimated by pooling all the data together (Section 2). However, this simple scenario rarely occurs in the real world. Provided that a dataset is large enough, virtually all populations are overdispersed with respect to the multinomial pointcounting uncertainties (Section 4).

For two-component systems, the degree of overdispersion can be visually assessed on a radial plot (Section 3). The dispersion may then be quantified using a three parameter continuous mixture model (Section 5), which can be generalised to three (or more) components (Section 6). The continuous mixture model assumes that the point-counting data are underlain by a logistic normal distribution. Although more realistic than the homogeneous population assumed by the pooled composition, the continuous mixture model is still a very simple approximation to real geological scenarios.

Correspondence Analysis was introduced as an effective tool for exploratory analysis of more complex and higher dimensional datasets (Section 7). It does not seek to capture the data in a simplified analytical form. Instead, CA distills the salient similarities and differences between samples as a two-dimensional 'map', and which the variables can also be shown. Such biplots can provide valuable geological insights that would be difficult to obtain otherwise.

CA is closely related to Principal Component Analysis. PCA can be applied to compositional data and uses Aitchison's Euclidean logratio-distance as a measure to compare the (dis)similarities between samples. In 
contrast, CA uses the chi-square distance (Equation 13), which makes it immune to the zero-count problem. Once we recognise the close affinity between the Aitchison distance and compositional data on the one hand, and between the chi-square distance and point-counting data on the other hand, then it is possible to add further complexity to our statistical analysis.

For example, Vermeesch and Garzanti (2015) introduce a technique called 3-way multidimensional scaling to combine different datasets together for the purpose of sedimentary provenance analysis. Using the insights gained from this paper, we could use the Aitchison distance to compare the major and trace element compositions of different samples, and the chi-square distance to compare their bulk petrography and heavy mineral counts.

\section{Acknowledgments}

The methods presented in this paper follow from the work of Rex Galbraith, who is gratefully acknowledged for patiently answering numerous questions from the author. Gert-Jan Weltje and an anonymous reviewer are thanked for their constructive comments on the submitted manuscript.

\section{References}

Aitchison, J. The statistical analysis of compositional data. Journal of the Royal Statistical Society, 44:139-177, 1982.

Aitchison, J. Principal component analysis of compositional data. Biometrika, 70(1):57-65, 1983. doi: $10.1093 /$ biomet/70.1.57.

Aitchison, J. The statistical analysis of compositional data. London, Chapman and Hall, 1986.

Aitchison, J. and Greenacre, M. Biplots of compositional data. Journal of the Royal Statistical Society: Series C (Applied Statistics), 51(4):375-392, 2002.

Barkley, F. A. The statistical theory of pollen analysis. Ecology, 15(3):283-289, 1934.

Berger, W. and Diester-Haass, L. Paleoproductivity: the benthic/planktonic ratio in foraminifera as a productivity index. Marine Geology, 81(1-4):15-25, 1988. 
Bloemsma, M. R. and Weltje, G. J. Reduced-rank approximations to spectroscopic and compositional data: A universal framework based on log-ratios and counting statistics. Chemometrics and Intelligent Laboratory Systems, 142:206-218, 2015.

Buzas, M. A. Another look at confidence limits for species proportions. Journal of Paleontology, 64(5):842-843, 1990.

Clark, R. L. Point count estimation of charcoal in pollen preparations and thin sections of sediments. Pollen et spores, 1982.

Dryden, A. Accuracy in percentage representation of heavy mineral frequencies. Proceedings of the National Academy of Sciences, 17(5):233-238, 1931.

Fatela, F. and Taborda, R. Confidence limits of species proportions in microfossil assemblages. Marine Micropaleontology, 45(2):169-174, 2002.

Galbraith, R. F. Statistics for fission track analysis. CRC Press, 2005.

Galbraith, R. Graphical display of estimates having differing standard errors. Technometrics, 30(3):271-281, 1988.

Galbraith, R. and Laslett, G. Statistical models for mixed fission track ages. Nuclear tracks and radiation measurements, 21(4):459-470, 1993.

Greenacre, M. Weighted metric multidimensional scaling. In New developments in classification and data analysis, pages 141-149. Springer, 2005.

Greenacre, M. J. Theory and applications of correspondence analysis. page 364, 1984.

Heroy, D. C., Kuehl, S. A., and Goodbred Jr, S. L. Mineralogy of the Ganges and Brahmaputra Rivers: implications for river switching and Late Quaternary climate change. Sedimentary Geology, 155(3-4):343$359,2003$.

Herzschuh, U. Reliability of pollen ratios for environmental reconstructions on the Tibetan Plateau. Journal of Biogeography, 34(7):1265-1273, 2007.

Kruskal, J. B. and Wish, M. Multidimensional scaling, volume 07-011 of Sage University Paper series on Quantitative Application in the Social Sciences. Sage Publications, Beverly Hills and London, 1978. 
Legendre, P. and Gallagher, E. D. Ecologically meaningful transformations for ordination of species data. Oecologia, 129(2):271-280, 2001.

Martín-Fernández, J. A., Barceló-Vidal, C., and Pawlowsky-Glahn, V. Dealing with zeros and missing values in compositional data sets using nonparametric imputation. Mathematical Geology, 35(3):253-278, 2003.

Morton, A. C. and Hallsworth, C. Identifying provenance-specific features of detrital heavy mineral assemblages in sandstones. Sedimentary Geology, 90(3-4):241-256, 1994.

Patterson, R. T. and Fishbein, E. Re-examination of the statistical methods used to determine the number of point counts needed for micropaleontological quantitative research. Journal of Paleontology, 63(2):245-248, 1989.

Pawlowsky-Glahn, V. and Buccianti, A. Compositional data analysis: Theory and applications. John Wiley \& Sons, 2011.

Pearson, K. On lines and planes of closest fit to systems of points in space. The London, Edinburgh, and Dublin Philosophical Magazine and Journal of Science, 2(11):559-572, 1901.

Van der Plas, L. and Tobi, A. A chart for judging the reliability of point counting results. American Journal of Science, 263(1):87-90, 1965.

Vermeesch, P. Multi-sample comparison of detrital age distributions. Chemical Geology, 341:140-146, 2013.

Vermeesch, P. and Garzanti, E. Making geological sense of 'Big Data' in sedimentary provenance analysis. Chemical Geology, 409:20-27, 2015.

Vermeesch, P., Resentini, A., and Garzanti, E. An R package for statistical provenance analysis. Sedimentary Geology, 2016.

Weltje, G. Quantitative analysis of detrital modes: statistically rigorous confidence regions in ternary diagrams and their use in sedimentary petrology. Earth-Science Reviews, 57(3-4):211 - 253, 2002. ISSN 0012-8252. doi: DOI: 10.1016/S0012-8252(01)00076-9.

Wendt, I. and Carl, C. The statistical distribution of the mean squared weighted deviation. Chemical Geology: Isotope Geoscience Section, 86(4):275-285, 1991. 
${ }_{449}$ Weng, C., Hooghiemstra, H., and Duivenvoorden, J. F. Challenges in estimating past plant diversity from ${ }_{450}$ fossil pollen data: statistical assessment, problems, and possible solutions. Diversity and distributions, 12(3): $451 \quad 310-318,2006$. 